\title{
Genetic variants account for up to one-third of cases of cerebral palsy
}

Recent exome sequencing evidence that reveals a genetic etiology in a substantial number of cerebral palsy cases challenges the continuing belief that birth asphyxia secondary to adverse intrapartum events is the disorder's leading cause

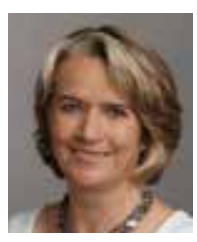

\section{Mary E. Norton, MD}

Professor of Obstetrics, Gynecology, and Reproductive Sciences

Director, Division of Maternal-Fetal Medicine

University of California, San Francisco

erebral palsy (CP) is the most common cause of severe neurodisability in children, and it occurs in about 2 to 3 per 1,000 births worldwide. ${ }^{1}$ This nonprogressive disorder is characterized by symptoms that include spasticity, dystonia, choreoathetosis, and/or ataxia that are evident in the first few years of life. While many perinatal variables have been associated with $\mathrm{CP}$, in most cases a specific cause is not identified.

Other neurodevelopmental disorders, such as intellectual disability, epilepsy, and autism spectrum disorder, are often associated with $\mathrm{CP}^{2}$ These other neurodevelopmental disorders are often genetic, and this has raised the question as to whether CP also might have a substantial genetic component, although this has not been investigated in any significant way until recently. This

The author reports no financial relationships relevant to this article. topic is of great interest to the obstetric community, given that $\mathrm{CP}$ often is attributed to obstetric events, including mismanagement of labor and delivery.

\section{Emerging evidence of a} genetic-CP association

In an article published recently in $J A M A$, Moreno-De-Luca and colleagues sought to determine the diagnostic yield of exome sequencing for $\mathrm{CP}^{3}$ This large cross-sectional study included results of exome sequencing performed in 2 settings. The first setting was a commercial laboratory in which samples were sent for analysis due to a diagnosis of $\mathrm{CP}$, primarily in children $(n=1,345)$ with a median age of 8.8 years. A second cohort, recruited from a neurodevelopmental disorders clinic at Geisinger, included primarily adults $(\mathrm{n}=181)$ with a median age of 41.9 years.

As is standard in exome sequencing, results were considered likely causative if they were classified as pathogenic or likely pathogenic based on criteria of the American CONTINUED ON PAGE 38 
College of Genetics and Genomics. In the laboratory group, $32.7 \%$ (440 of 1,345 ) had a genetic cause of the CP identified, while in the clinic group, $10.5 \%$ (19 of 181) had a genetic etiology found. Although most of the identified genetic variants were de novo (that is, they arose in the affected individual and were not clearly inherited), some were inherited from carrier parents. ${ }^{3}$

A number of other recent studies also have investigated genetic causes of $\mathrm{CP}$ and similarly have reported that a substantial number of cases are genetic. Several studies that performed chromosomal microarray analysis in individuals with CP found deleterious copy number variants in $10 \%$ to $31 \%$ of cases. ${ }^{4-6}$ Genomic variants detectable by exome sequencing have been reported in $15 \%$ to $20 \%$ of cases. ${ }^{3}$ In a recent study in Nature Genetics, researchers performed exome sequencing on 250 parentchild "trios" in which the child had $\mathrm{CP}$, and they found that $14 \%$ of cases had an associated genetic variant that was thought to be causative. ${ }^{4}$ These studies all provide consistent evidence that a substantial proportion of CP cases are due to genetic causes.

\section{Contributors to CP risk}

Historically, CP was considered to occur largely as a result of perinatal anoxia. In 1862, the British orthopedic surgeon William John Little first reported an association between prematurity, asphyxia, difficult delivery, and $\mathrm{CP}$ in a paper presented to the Obstetrical Society of London. ${ }^{7}$ Subsequently, much effort has gone into the prevention of perinatal asphyxia and birth injury, although our ability to monitor fetal well-being remains limited. Nonreassuring fetal heart rate patterns are nonspecific and can occur for many reasons other than fetal asphyxia. Studies of electronic fetal monitoring have found that continuous monitoring primarily leads to an increase in cesarean delivery with no decrease in $\mathrm{CP}$ or infant mortality. ${ }^{8}$

While some have attributed this to failure to accurately interpret the fetal heart rate tracing, it also may be because a substantial number of $\mathrm{CP}$ cases are due to genetic and other causes, and that very few in fact result from preventable intrapartum injury.

The American College of Obstetricians and Gynecologists and the American Academy of Pediatrics agree that knowledge gaps preclude definitive determination that a given case of neonatal encephalopathy is attributable to an acute intrapartum event, and they provide criteria that must be fulfilled to establish a reasonable causal link between an intrapartum event and subsequent long-term neurologic disability. ${ }^{9}$ However, there continues to be a belief in the medical, scientific, and lay communities that birth asphyxia, secondary to adverse intrapartum events, is the leading cause of CP. A "brain-damaged infant" remains one of the most common malpractice claims, and birth injury one of the highest paid claims. Such claims generally allege that intrapartum asphyxia has caused long-term neurologic sequelae, including CP.

While it is true that prematurity, infection, hypoxia-ischemia, and pre- and perinatal stroke all have been implicated as contributing to $\mathrm{CP}$ risk, large populationbased studies have shown that birth asphyxia accounts for less than $12 \%$ of CP cases. $^{10}$ Specifically, recent data indicate that acute intrapartum hypoxia-ischemia occurs only in about $6 \%$ of CP cases. In other words, it does occur and may contribute to some cases, but this is likely a smaller percent than previously thought, and genetic factors now appear to be far more significant contributors. ${ }^{11}$

\section{Exploring a genetic etiology}

In considering the etiologies of $\mathrm{CP}$, it is important to note that $21 \%$ to $40 \%$ of individuals with CP have an associated congenital anomaly, suggesting a genetic origin in at least some individuals. Moreover, a $40 \%$ heritability has been estimated in $\mathrm{CP}$, which is comparable to the heritability rate for autism spectrum disorders. ${ }^{12}$

In the recent study by MorenoDe-Luca and colleagues, some of the gene variants detected were previously associated with other forms of neurodevelopmental disability, such as epilepsy and autism spectrum disorder. ${ }^{3}$ Many individuals in the study cohort were found to have multiple neurologic comorbidities, for example, CP as well as epilepsy, autism spectrum disorder, and/or intellectual disability. The presence of these additional comorbidities increased the likelihood of finding a genetic cause; the authors found that the diagnostic yield ranged from $11.2 \%$ with isolated CP to $32.9 \%$ with all 3 comorbidities. The yield was highest with $\mathrm{CP}$ and intellectual disability and CP with all 3 comorbidities. A few genes were particularly common, and some were reported previously in association with CP and/or other neurodevelopmental disorders. In some patients, variants were found in genes or gene regions associated with disorders that do not frequently include CP, such as Rett syndrome. ${ }^{3}$

\section{Implications for ObGyns}

The data from the study by MorenoDe-Luca and colleagues are interesting and relevant to pediatricians, 
neurologists, and geneticists, as well as obstetricians. Understanding the cause of any disease or disorder improves care, including counseling regarding the cause, the appropriate interventions or therapy, and in some families, the recurrence risk in another pregnancy. The treatment for CP has not changed significantly in many years. Increasingly, detection of an underlying genetic cause can guide precision treatments; thus, the detection of specific gene variants allows a targeted approach to therapy.

Identification of a genetic cause also can significantly impact recurrence risk counseling and prenatal diagnosis options in another pregnancy. In general, the empiric recurrence risk of $\mathrm{CP}$ is quoted as $1 \%$ to $2 \%,{ }^{13}$ and with de novo variants this does not change. However, with inherited variants the recurrence risk in future children is substantially higher. While $72 \%$ of the genetic variants associated with $\mathrm{CP}$ in the Moreno-De-Luca study were de novo with a low recurrence risk, in the other $28 \%$ the mode of inheritance indicated a substantial risk of recurrence (25\%-50\%) in another pregnancy. ${ }^{3}$ Detecting such causative variant(s) allows not only accurate counseling about recurrence risk but also preimplantation genetic testing or prenatal diagnosis when recurrence risk is high.

In the field of obstetrics, the debate about the etiology of CP is important largely due to the medicolegal implications. Patient-oriented information on the internet often states that CP is caused by damage to the child's brain just before, during, or soon after birth, supporting potential blame of those providing care during those times. Patient-oriented websites regarding CP do not list genetic disorders among the causes but rather include primarily environmental factors, such as prematurity, low birth weight, in utero infections, anoxia or other brain injury, or perinatal stroke. Even the Centers for Disease Control and Prevention website lists brain damage as the primary etiology of $\mathrm{CP}^{14}$ Hopefully, these new data will increase a broader understanding of this condition.

Exome sequencing is now recommended as a first-tier test for individuals with many neurodevelopmental disorders, including epilepsy, intellectual disability, and autism spectrum disorder. ${ }^{15}$ However, comprehensive genetic testing is not typically recommended or performed in cases of CP. Based on recent data, including the report by Moreno-De-Luca and colleagues, it would seem that CP should be added to the list of disorders for which exome sequencing is ordered, given the similar prevalence and diagnostic yield.
References

1. Oskoui M, Coutinho F, Dykeman J, et al. An update on the prevalence of cerebral palsy: a systematic review and meta-analysis. Dev Med Child Neurol. 2013;55:509-519.

2. Rosenbaum P, Paneth N, Leviton A, et al. A report: the definition and classification of cerebral palsy April 2006. Dev Med Child Neurol Suppl. 2007;109:8-14.

3. Moreno-De-Luca A, Millan F, Pesacreta DR, et al. Molecular diagnostic yield of exome sequencing in patients with cerebral palsy. JAMA. 2021;325:467-475.

4. Jin SC, Lewis SA, Bakhtiari S, et al. Mutations disrupting neuritogenesis genes confer risk for cerebral palsy. Nat Genet. 2020;52:1046-1056.

5. Segel R, Ben-Pazi H, Zeligson S, et al. Copy number variations in cryptogenic cerebral palsy. $\mathrm{Neu}$ rology. 2015;84:1660-1668.

6. McMichael G, Girirrajan S, Moreno-De-Luca A, et al. Rare copy number variation in cerebral palsy. Eur J Hum Genet. 2014;22:40-45.
7. Little WJ. On the influence of abnormal parturition, difficult labours, premature births, and asphyxia neonatorum, on the mental and physical condition of the child, especially in relation to deformities. Trans Obstet Soc Lond. 1862;3:293-344.

8. Alfirevic Z, Devane D, Gyte GM. Continuous cardiotocography (CTG) as a form of electronic fetal monitoring (EFM) for fetal assessment during labour. Cochrane Database Syst Rev. 2013;5;CD006066.

9. American College of Obstetricians and Gynecologists. Executive summary: neonatal encephalopathy and neurologic outcome second edition. Report of the American College of Obstetricians and Gynecologists' Task Force on Neonatal Encephalopathy. Obstet Gynecol. 2014;123:896901.

10. Ellenberg JH, Nelson KB. The association of cerebral palsy with birth asphyxia: a definitional quagmire. Dev Med Child Neurol. 2013;55:210216.
11. Himmelmann K, Uvebrant P. The panorama of cerebral palsy in Sweden part XII shows that patterns changed in the birth years 2007-2010. Acta Paediatr. 2018;107: 462-468.

12. Petterson B, Stanley F, Henderson D. Cerebral palsy in multiple births in Western Australia: genetic aspects. Am J Med Genet. 1990;37:346351.

13. Korzeniewski SJ, Slaughter J, Lenski M, et al. The complex aetiology of cerebral palsy. Nat Rev Neurol. 2018;14:528-543.

14. Centers for Disease Control and Prevention. Causes and risk factors of cerebral palsy. https:// www.cdc.gov/ncbddd/cp/causes.html. Accessed March 23, 2021.

15. Srivastava S, Love-Nichols JA, Dies KA, et al; NDD Exome Scoping Review Work Group. Meta-analysis and multidisciplinary consensus statement: exome sequencing is a first-tier clinical diagnostic test for individuals with neurodevelopmental disorders. Genet Med. 2019;21:2413-2421. 